\title{
Numerical Investigation of Forced Convection in a Channel with Solid Block inside a Square Porous Block
}

\author{
Neda Janzadeh and Mojtaba Aghajani Delavar \\ Islamic Azad University Science and Research Ayatollah Amoli Branch, Amol, Iran \\ Correspondence should be addressed to Mojtaba Aghajani Delavar; m.a.delavar@nit.ac.ir
}

Received 13 February 2013; Accepted 7 April 2013

Academic Editor: S. A. Kalogirou

Copyright (c) 2013 N. Janzadeh and M. Aghajani Delavar. This is an open access article distributed under the Creative Commons Attribution License, which permits unrestricted use, distribution, and reproduction in any medium, provided the original work is properly cited.

\begin{abstract}
The fluid flow and heat transfer in a porous medium have received considerable attention due to its importance in many engineering applications. In this study numerical investigation of fluid flow and heat transfer over a hot solid block inside a square porous block located in a channel was carried out. The lattice Boltzmann method with nine velocities, D2Q9, was used for numerical simulations. Brinkman-Forchheimer model was successfully used to simulate fluid flow in porous media. The effects of parameters such as porosity, Reynolds number on flow pattern, and heat transfer were studied. The different effects of mentioned parameters were discussed in the paper.
\end{abstract}

\section{Introduction}

The fluid flow and transport phenomena in a porous medium have been used in many fields of science and engineering, such as geothermal energy extraction, solar collector, solar absorbers food processing, fuel cells, petroleum processing, catalytic and chemical particle beds, transpiration cooling, electronic cooling, drying processes, porous bearing, nuclear reactors, and many others.

Thermal Insulation. Fluid flow and convective heat transfer in conduits fully and partially filled with porous medium have been investigated analytically, experimentally, and numerically by many researchers. Poulikakos and Kazmierczak [1] theoretically studied the convection heat transfer in the developed region of two parallel plates and in a circular pipe with porous media attached to the wall. Fu et al. [2] numerically simulated the convection heat transfer through a single porous block mounted on the heated wall, and they studied the effect of different parameters including the porous block size and length and porosity of porous block and Reynolds number on convection heat transfer enhancement. Li et al. [3] numerically investigated the effect of staggered porous blocks mounted on the wall in a laminar flow channel on the velocity field and local heat transfer. Huang et al.
[4] numerically studied steady-state heat transfer to a fluid passing through multiple heated block mounted on the wall of a channel with porous covers.

Guo et al. [5] investigated numerically the heat transfer of pulsating flow through a pipe with a porous medium attached to the pipe wall. In their work they used conventional control volume method. Hamdan et al. [6] used an implicit finitedifference method to simulate forced convection in a porous substrate inserted in the core of a parallel plate channel. Jiang et al. [7] investigate the forced convection heat transfer in plate channels saturated with air and water which contain sintered bronze porous media.

The lattice Boltzmann method (LBM) is a powerful numerical technique based on kinetic theory for simulation of fluid flows and modeling the physics in fluids [8-10]. The problem of convective heat in porous medium was investigated numerically by Guo and Zhao [11]. In their work, they used the lattice Boltzmann method to simulate the temperature field. Seta et al. [12] applied the lattice Boltzmann method to simulation of natural convection in porous media. Shokouhmand et al. [13] simulated the laminar flow and convective heat transfer in a porous medium inside the core of two parallel plates of a conduit using lattice Boltzmann method. 
Hot solid block inside a square porous block may be applied in some engineering application such as porous media as an active layer in reacting chemical flows, fuel cell, heat exchangers, and electronic devices cooling.

\section{Numerical Model}

The general form of lattice Boltzmann equation with nine velocities, D2Q9, with external force can be written as [9]

$$
\begin{aligned}
& f_{k}\left(\vec{x}+\vec{c}_{k} \Delta t, t+\Delta t\right)= f_{k}(\vec{x}, t) \\
&+\frac{\Delta t}{\tau}\left[f_{k}^{\mathrm{eq}}(\vec{x}, t)-f_{k}(\vec{x}, t)\right]+\Delta t \vec{F}_{k}, \\
& f_{k}^{\mathrm{eq}}=\omega_{k} \cdot \rho\left[1+\frac{\vec{c}_{k} \cdot \vec{u}}{c_{s}^{2}}+\frac{1}{2} \frac{\left(\vec{c}_{k} \cdot \vec{u}\right)^{2}}{c_{s}^{4}}-\frac{1}{2} \frac{\vec{u} \cdot \vec{u}}{c_{s}^{2}}\right],
\end{aligned}
$$

where $\vec{c}_{k}$ is the discrete lattice velocity in direction $k, \vec{F}_{k}$ is the external force, $\Delta t$ denotes the lattice time step, $\tau$ is the lattice relaxation time, $f_{k}^{\text {eq }}$ denotes the equilibrium distribution function, $\omega_{k}$ is weighting factor, and $\rho$ is the lattice fluid density. To consider both the flow and the temperature fields, the thermal LBM utilizes two distribution functions, $f$ and $g$, for flow and temperature fields, respectively. The $f$ distribution function is as same as discussed previously; the $g$ distribution function is as follows [9]:

$$
\begin{aligned}
& g_{k}\left(\vec{x}+\vec{c}_{k} \Delta t, t+\Delta t\right)= g_{k}(\vec{x}, t) \\
&+\frac{\Delta t}{\tau_{g}}\left[g_{k}^{\mathrm{eq}}(\vec{x}, t)-g_{k}(\vec{x}, t)\right], \\
& g_{k}^{\mathrm{eq}}=\omega_{k} \cdot T \cdot\left[1+\frac{\vec{c}_{k} \cdot \vec{u}}{c_{s}^{2}}\right] .
\end{aligned}
$$

The flow properties are defined as ( $i$ denotes the component of the Cartesian coordinates)

$$
\rho=\sum_{k} f_{K}, \quad \rho u_{i}=\sum_{k} f_{k} c_{k i}, \quad T=\sum_{k} g_{k} .
$$

The Brinkman-Forchheimer equation was used for flow in porous regions that is written as $[9,12]$

$$
\begin{aligned}
\frac{\partial \vec{u}}{\partial t}+(\vec{u} \cdot \nabla)\left(\frac{\vec{u}}{\varepsilon}\right)= & -\frac{1}{\rho} \nabla(\varepsilon p)+v_{\text {eff }} \nabla^{2} \vec{u} \\
& +\left(-\frac{\varepsilon v}{K} \vec{u}-\frac{1.75}{\sqrt{150 \varepsilon K}}|\vec{u}| \vec{u}+\varepsilon \vec{G}\right),
\end{aligned}
$$

where $\varepsilon$ is the porosity, $K$ is the permeability, $v_{\text {eff }}$ is the effective viscosity, $v$ is the kinematic viscosity, and $G$ is the acceleration due to gravity. The last term in the right hand in the parenthesis is the total body force, $F$, which was written by using the widely used Ergun's relation [16]. For porous medium the corresponding distribution functions are as the same as (1). But the equilibrium distribution functions and the best choice for the forcing term are

$$
\begin{gathered}
f_{k}^{\mathrm{eq}}=\omega_{k} \cdot \rho \cdot\left[1+\frac{\vec{c}_{k} \cdot \vec{u}}{c_{s}^{2}}+\frac{1}{2} \frac{\left(\vec{c}_{k} \cdot \vec{u}\right)^{2}}{\varepsilon c_{s}^{4}}-\frac{1}{2} \frac{\vec{u}^{2}}{\varepsilon c_{s}^{2}}\right], \\
F_{k}=\omega_{k} \rho\left(1-\frac{1}{2 \tau_{v}}\right)\left[\frac{\vec{c}_{k} \cdot \vec{F}}{c_{s}^{2}}+\frac{\left(\vec{u} \vec{F}: \vec{c}_{k} \vec{c}_{k}\right)}{\varepsilon c_{s}^{4}}-\frac{\vec{u} \cdot \vec{F}}{\varepsilon c_{s}^{2}}\right] .
\end{gathered}
$$

The forcing term $F_{k}$ defines the fluid velocity $\vec{u}$ as

$$
\vec{u}=\sum_{k} \frac{c_{k} F_{k}}{\rho}+\frac{\Delta t}{2} \vec{F}
$$

According the previous equations $\vec{F}$ is related to $\vec{u}$, so (6) is nonlinear for the velocity. A temporal velocity $\vec{v}$ is used to solve this nonlinear problem [13] as follows:

$$
\begin{array}{rlrl}
\vec{u} & =\frac{\vec{v}}{c_{0}+\sqrt{c_{0}^{2}+c_{1}|\vec{v}|}}, & \vec{v} & =\sum_{k} \frac{c_{k} f_{k}}{\rho}+\frac{\Delta t}{2}, \\
c_{0}=\frac{1}{2}\left(1+\varepsilon \frac{\Delta t}{2} \frac{v}{K}\right), & c_{1}=\varepsilon \frac{\Delta t}{2} \frac{1.75}{\sqrt{150 \varepsilon^{3} K}} .
\end{array}
$$

The effective thermal conductivity, $k_{\text {eff }}$ of the porous medium should be recognized for proper investigation of conjugate convection and conduction heat transfer in porous zone, which was calculated by [16]

$$
\begin{aligned}
k_{\mathrm{eff}}=k_{f}[ & (1-\sqrt{1-\varepsilon})+\frac{2 \sqrt{1-\varepsilon}}{1-\sigma B} \\
\times & \left.\left(\frac{(1-\sigma) B}{(1-\sigma B)^{2}} \ln \left(\frac{1}{\sigma B}\right)-\frac{B+1}{2}-\frac{B-1}{1-\sigma B}\right)\right], \\
B & =1.25\left[\frac{1-\varepsilon}{\varepsilon}\right]^{10 / 9}, \quad \sigma=\frac{k_{f}}{k_{s}} .
\end{aligned}
$$

\section{Boundary Conditions}

From the streaming process the distribution functions out of the domain are known. The unknown distribution functions are those toward the domain. In Figure 1 the unknown distribution function which needs to be determined, are shown as dotted lines. Regarding the boundary conditions of the flow field, the solid walls are assumed to be no slip, and thus the bounce-back scheme is applied. This scheme specifies the outgoing directions of the distribution functions as the reverse of the incoming directions at the boundary sites. For example, for flow field in the north boundary the following conditions is used:

$$
f_{4, n}=f_{2, n}, \quad f_{7, n}=f_{5, n}, \quad f_{8, n}=f_{6, n} .
$$

In this channel the inlet velocity at west boundary is known $u=u_{w}=u_{\text {inlet. }}$. In LBM method the inward distribution functions at the boundaries have to be specified. So the 


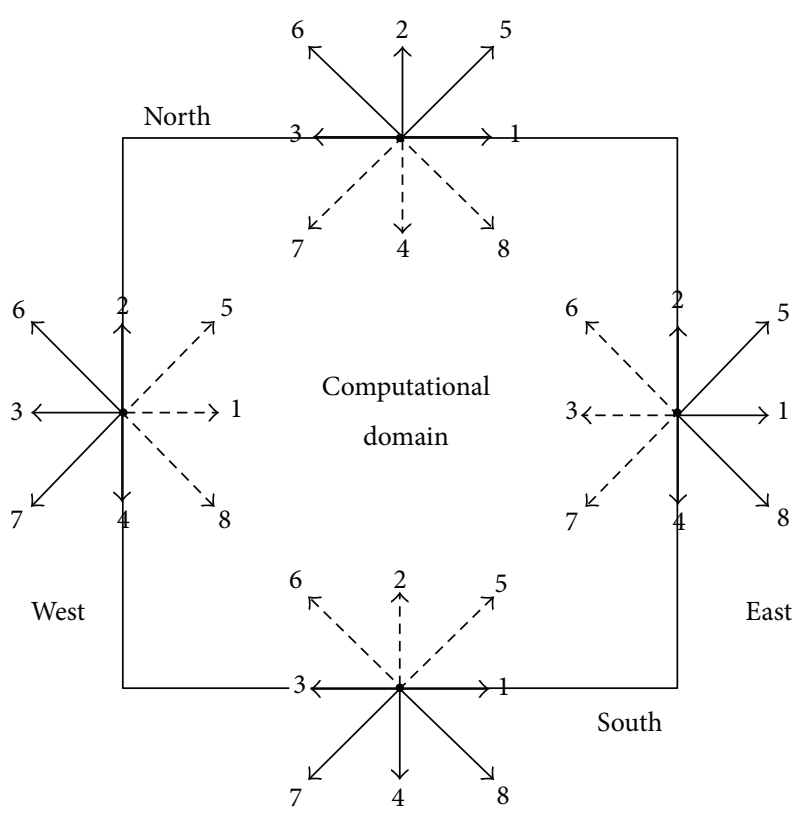

FIgure 1: Domain boundaries and known (solid lines) and unknown (dotted lines) distribution functions.

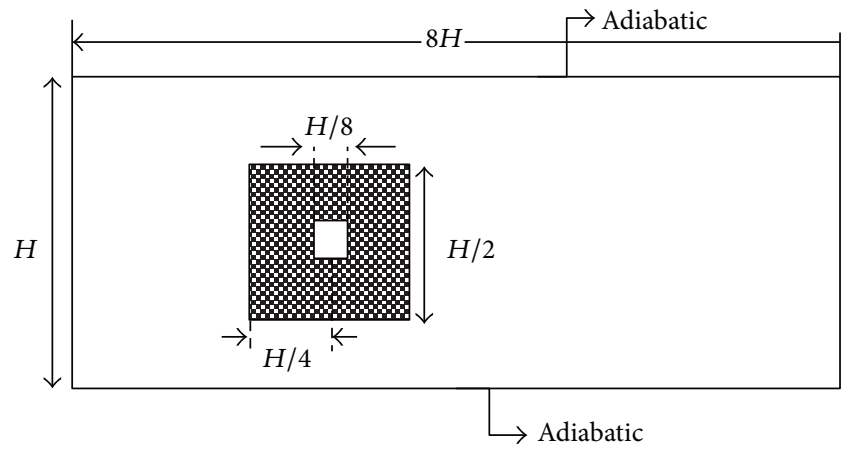

Figure 2: The computational domain.

$f_{1}, f_{5}$, and $f_{8}$ are unknowns for west boundary condition. The unknown distribution functions are calculated as

$$
\begin{gathered}
\rho_{w}=\frac{1}{1-u_{w}}\left[f_{0}-f_{2}+f_{4}+2\left(f_{3}+f_{6}+f_{7}\right)\right] \\
f_{1, n}=f_{3, n}+\frac{2}{3} \rho_{w} \quad f_{5, n}=f_{7, n}-\frac{1}{2}\left(f_{2, n}-f_{4, n}\right)+\frac{1}{6} \rho_{w} u_{w}, \\
f_{8, n}=f_{6, n}+\frac{1}{2}\left(f_{2, n}-f_{4, n}\right)+\frac{1}{6} \rho_{w} u_{w} .
\end{gathered}
$$

In this problem the outlet velocity is unknown. The east boundary condition in Figure 1 represents the outlet condition. Then $f_{3}, f_{6}$, and $f_{7}$ need to be calculated at the east boundary as

$$
\begin{gathered}
f_{3, n}=2 f_{3, n-1}-f_{3, n-2}, \quad f_{6, n}=2 f_{6, n-1}-f_{6, n-2}, \\
f_{7, n}=2 f_{7, n-1}-f_{7, n-2} .
\end{gathered}
$$

TABLE 1: Simulation parameters.

\begin{tabular}{lc}
\hline$L$ & $8.0 \mathrm{~cm}$ \\
$\varepsilon$ & $0.3-0.5-0.7-0.9$ \\
$\operatorname{Pr}$ & 0.7 \\
$H$ & $1.0 \mathrm{~cm}$ \\
$\operatorname{Re}$ & $25-50-75-100$ \\
$T_{\text {inlet }}-T_{\text {wall solid block }}$ & $20^{\circ} \mathrm{C}-40^{\circ} \mathrm{C}$ \\
\hline
\end{tabular}

TABLE 2: Comparison of averaged Nusselt number between LBM and Kays and Crawford [17].

\begin{tabular}{lccc}
\hline & & $q_{2}^{\prime \prime} / q_{1}^{\prime \prime}$ & \\
& 0.5 & 1.0 & 1.5 \\
\hline $\mathrm{Nu}_{1}$ & & & \\
$\quad$ Kays and Crawford [17] & 17.48 & 8.23 & 11.19 \\
$\quad$ LBM & 17.25 & 8.16 & 11.10 \\
$\mathrm{Nu}_{2}$ & & & \\
$\quad$ Kays and Crawford [17] & 6.51 & 8.23 & 7.00 \\
$\quad$ LBM & 6.49 & 8.16 & 6.91 \\
\hline
\end{tabular}

Furthermore, for the temperature field, the local temperature is defined by (3). For isothermal boundaries such as a bottom hot wall the unknown distribution functions are evaluated as

$$
\begin{gathered}
g_{2, n}=T_{h}\left(\omega_{2}+\omega_{4}\right)-g_{4, n}, \quad g_{5, n}=T_{h}\left(\omega_{5}+\omega_{7}\right)-g_{7, n}, \\
g_{6, n}=T_{h}\left(\omega_{6}+\omega_{8}\right)-g_{8, n},
\end{gathered}
$$

where $n$ is the lattice on the boundary. For adiabatic boundary condition such as a bottom wall the unknown distribution functions are evaluated as

$$
g_{2, n}=g_{2, n-1}, \quad g_{5, n}=g_{5, n-1}, \quad g_{6, n}=g_{6, n-1} .
$$

\section{Computational Domain}

Computational domain consists of a hot solid block inside a square porous block located in a channel (Figure 2). The channel height, length, and other simulation parameters are illustrated in the Figure 2 and Table 1.

\section{Validation and Grid Independent Check}

In this study, flow pattern and thermal field were simulated in a channel with a hot solid block inside a porous block by using lattice Boltzmann method. Figure 3 compares well the velocity profile for flow in a clear channel captured by LBM and results presented by Nield [14]. In this figure the result of simulation is compared well with Seta et al. [15]. Table 2 shows the computed Nusselt number by LBM and Kays and Crawford [17], and good agreement is obtained.

\section{Results and Discussion}

In the present work the thermal lattice Boltzmann model with nine velocities was used to solve the force convection 


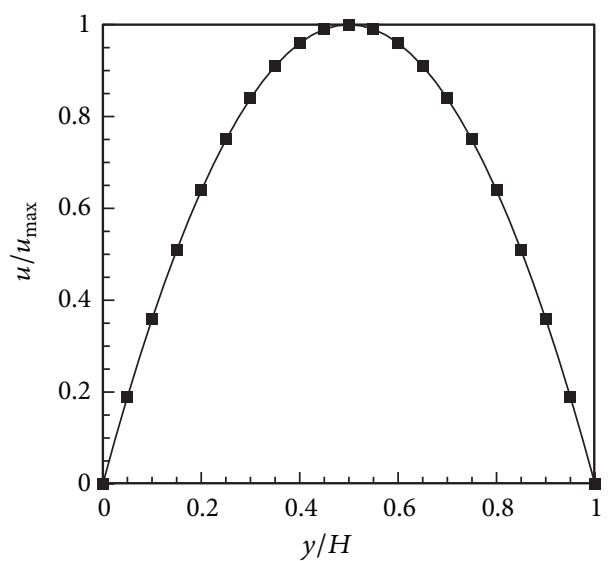

- Nield (2004)

- Present study

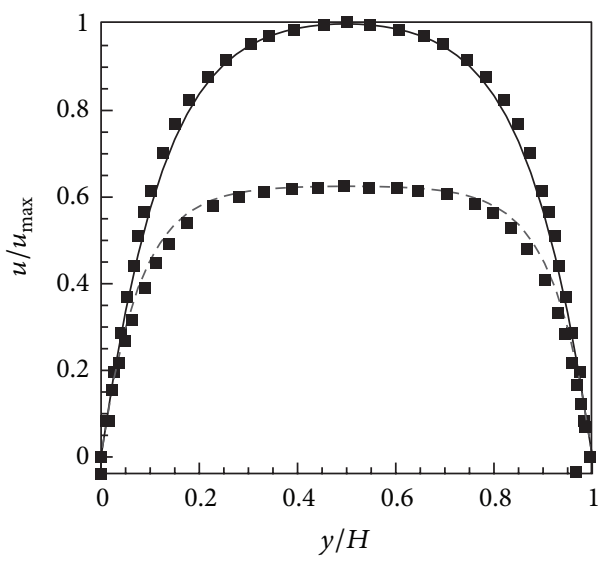

- Present study $(\mathrm{Re}=10)$

- - Present study $(\operatorname{Re}=1)$

- Seta et al. (2006)

FIGURE 3: Comparison of velocity profile for clear channel between Nield [14] and LBM and porous channel flow between LBM and Seta et al. [15].
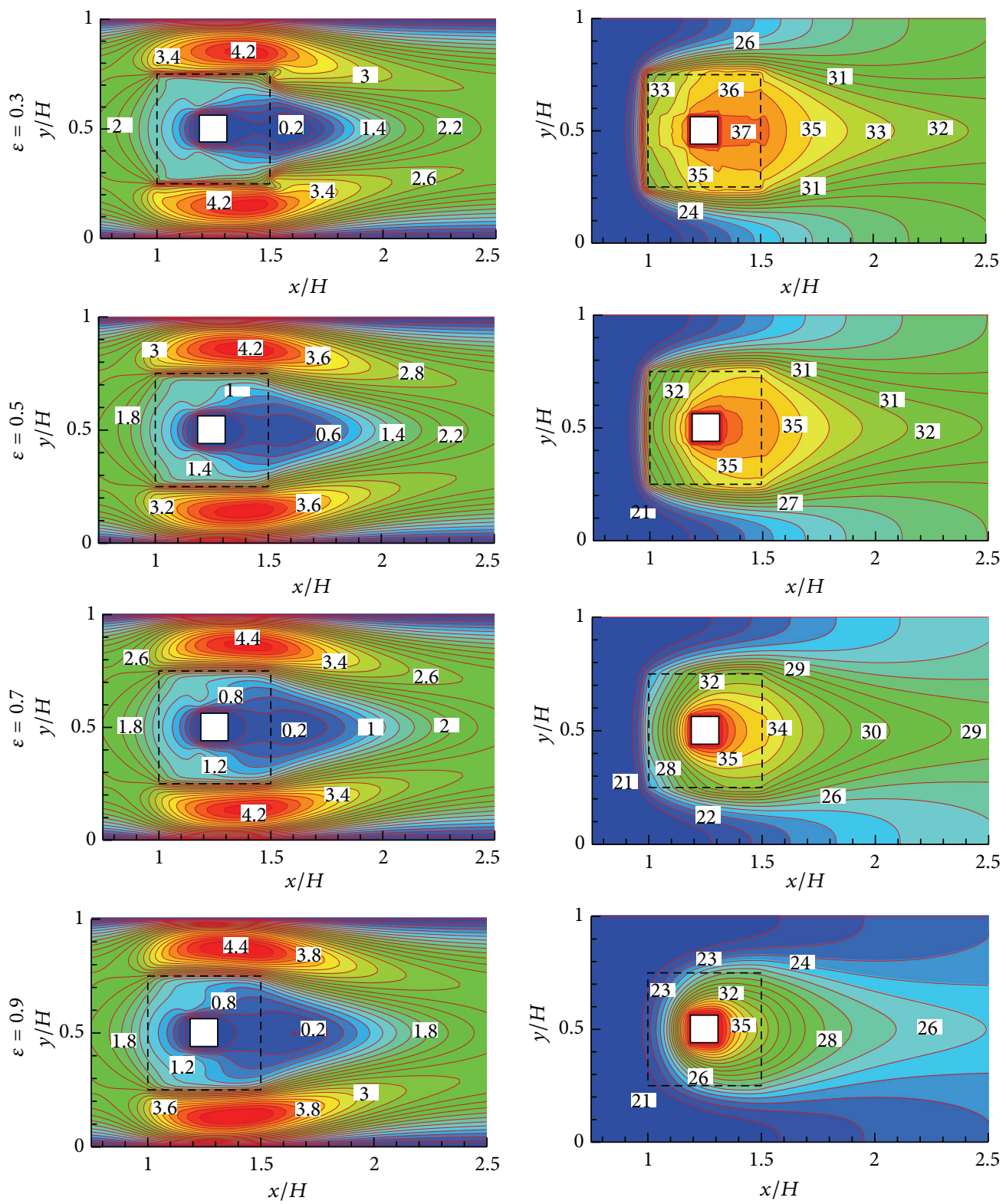

FIGURE 4: Velocity contours $\left(\mathrm{U} / \mathrm{U}_{\text {inlet }(\mathrm{Re}=25)}\right)$ temperature contours for different porosities. 

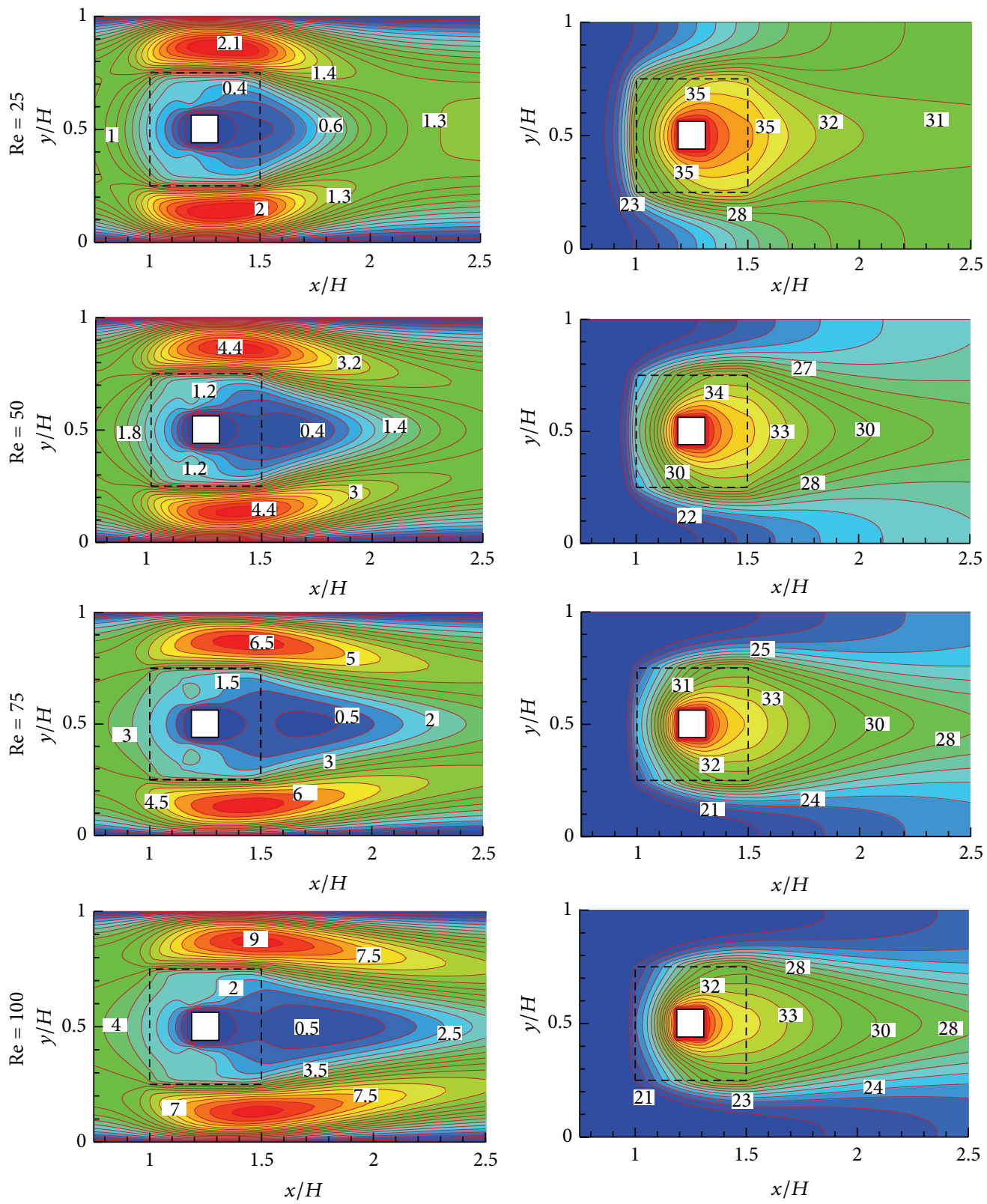

FIGURE 5: Velocity vectors and contours $\left(\mathrm{U} / \mathrm{U}_{\text {inlet }(\mathrm{Re}=25)}\right)$ for different Reynolds numbers.

heat transfer in a channel containing hot solid block inside a square porous block. The effects of porosity and Reynolds number, on the flow field and convective heat transfer, were investigated.

Figure 4 shows the velocity and temperature contours for different values of porosity at $\mathrm{Re}=50$. With increasing the porosity, velocity rises slightly in the top and bottom of porous block, because fluid tends to flow in clear passages with lower pressure drop. At higher values of porosity, fluid changes easier its path to clear passages, so velocity increases in these passages.

According to (8) with increasing the porosity, the effective thermal conductivity in the porous block reduces. At higher value of porosity, the heat transfer between fluid and solid block decreases. So the fluid temperature will increase.
The velocity and temperature contours for different Reynolds number have been drown in Figure 5.

It can be seen that as Reynolds number increases the flow velocity increases. With increasing the velocity, the convective coefficient $(h)$ increased according to $\left(Q=h A\left(T_{\text {wall }}-\right.\right.$ $\left.T_{\text {fluid }}\right)$ ), and the magnitude of heat transfer increases. But According to $\left(Q=m c \Delta T=\rho u A\left(T_{\text {out }}-T_{\text {in }}\right)\right)$ with increasing the velocity, the temperature gradient of the fluid reduces. So the outlet fluid temperature will decrease.

The temperature and velocity profiles for various Reynolds numbers are represented in Figure 6. As expected the flow velocity increases with increasing the Reynolds number. The higher values of Reynolds number cause the temperature of zones to be decreased due to the decrease in heat transfer process. In Figure 7 the average fluid 


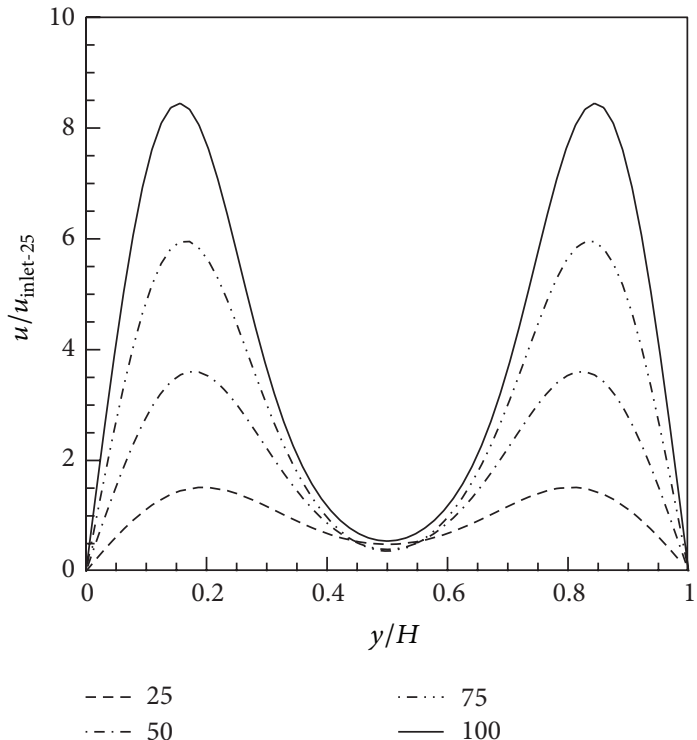

(a)

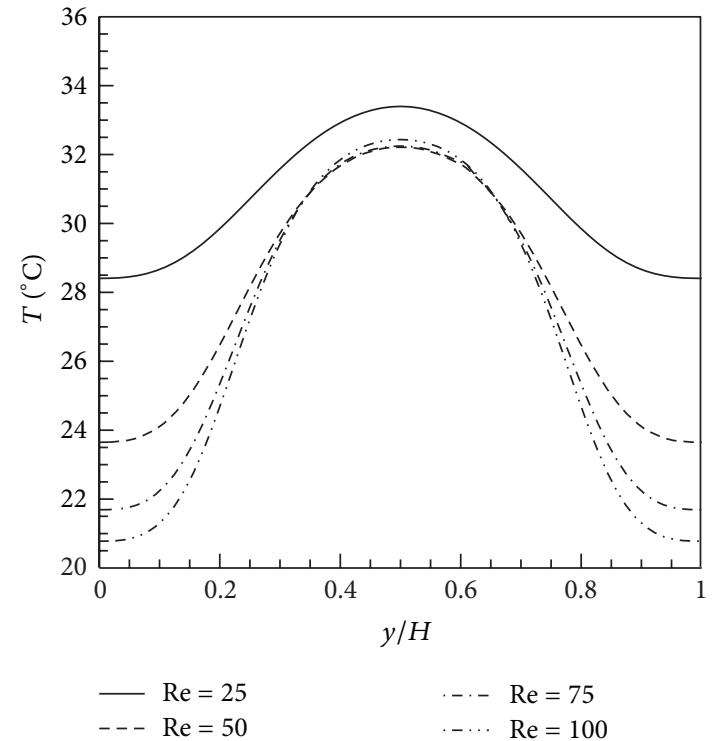

(b)

FIGURE 6: velocity and temperature profiles at different Reynolds numbers (porosity $=0.7, x / H=7 / 16$ ).

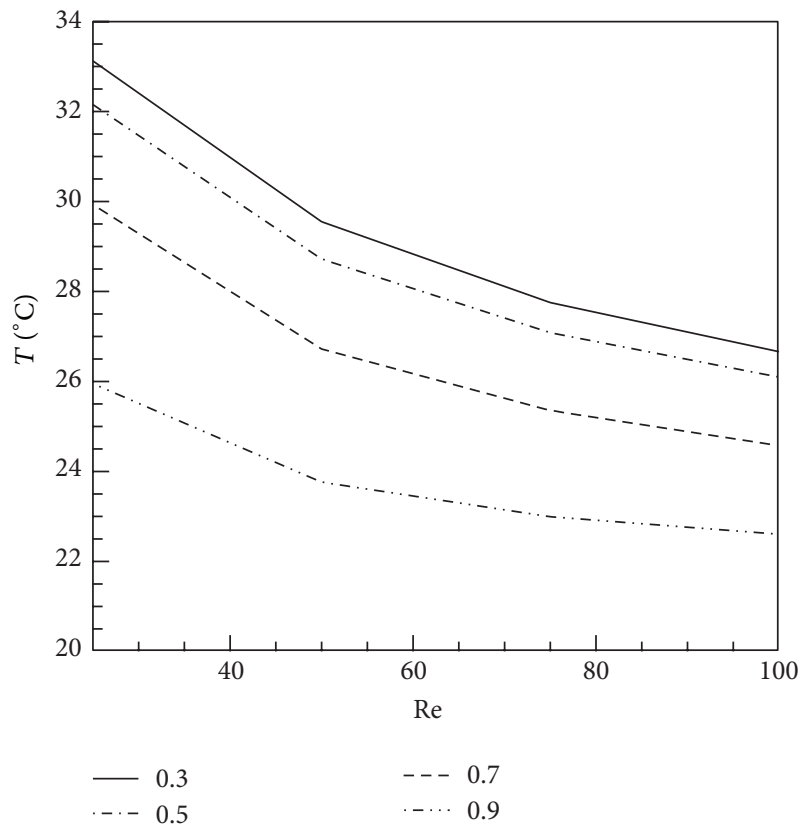

FIGURE 7: Averaged fluid temperature at different porosities.

temperature for various Reynolds numbers at different porosity is drown that shows the effect of Reynolds number on temperature more than porosity. As mentioned before at higher porosity obtains a lower average temperature for fluid.

\section{Conclusion}

Fluid flow and heat transfer in a channel with solid block inside a square porous block were carried out in this paper.
The effect of parameters including porosity and Reynolds number on the velocity and thermal field was investigated numerically by using lattice Boltzmann method. Decreasing the porosity leads to a higher temperature of fluid due to increasing $K_{\text {eff }}$ which causes the heat transfer enhancement in porous block. With increasing the Reynolds number the fluid velocity increased, and the average fluid temperature will be reduced.

\section{References}

[1] D. Poulikakos and M. Kazmierczak, "Forced convection in a duct partially filled with a porous material," Journal of Heat Transfer, vol. 109, no. 3, pp. 653-662, 1987.

[2] W. S. Fu, H. C. Huang, and W. Y. Liou, “Thermal enhancement in laminar channel flow with a porous block," International Journal of Heat and Mass Transfer, vol. 39, no. 10, pp. 2165-2175, 1996.

[3] H. Y. Li, K. C. Leong, L. W. Jin, and J. C. Chai, "Analysis of fluid flow and heat transfer in a channel with staggered porous blocks," International Journal of Thermal Sciences, vol. 49, no. 6 , pp. 950-962, 2010.

[4] P. C. Huang, C. F. Yang, J. J. Hwang, and M. T. Chiu, "Enhancement of forced-convection cooling of multiple heated blocks in a channel using porous covers," International Journal of Heat and Mass Transfer, vol. 48, no. 3-4, pp. 647-664, 2005.

[5] Z. Guo, S. Y. Kim, and H. J. Sung, "Pulsating flow and heat transfer in a pipe partially filled with a porous medium," International Journal of Heat and Mass Transfer, vol. 40, no. 17, pp. 4209-4218, 1997.

[6] M. O. Hamdan, M. A. Al-Nimr, and M. K. Alkam, "Enhancing forced convection by inserting porous substrate in the core of a parallel-plate channel," International Journal of Numerical Methods for Heat and Fluid Flow, vol. 10, no. 5, pp. 502-517, 2000.

[7] P. X. Jiang, M. Li, T. J. Lu, L. Yu, and Z. P. Ren, "Experimental research on convection heat transfer in sintered porous plate 
channels," International Journal of Heat and Mass Transfer, vol. 47, no. 10-11, pp. 2085-2096, 2004.

[8] S. Succi, The Lattice Boltzmann Equation for Fluid Dynamics and Beyond, Clarendon Press, Oxford, UK, 2001.

[9] A. A. Mohammad, Lattice Boltzmann Method, Fundamentals and Engineering Applications with Computer Codes, Springer, London, UK, 2011.

[10] P. H. Kao, Y. H. Chen, and R. J. Yang, "Simulations of the macroscopic and mesoscopic natural convection flows within rectangular cavities," International Journal of Heat and Mass Transfer, vol. 51, no. 15-16, pp. 3776-3793, 2008.

[11] Z. Guo and T. S. Zhao, "A lattice Boltzmann model for convection heat transfer in porous media," Numerical Heat Transfer, Part B, vol. 47, no. 2, pp. 157-177, 2005.

[12] T. Seta, E. Takegoshi, and K. Okui, "Lattice Boltzmann simulation of natural convection in porous media," Mathematics and Computers in Simulation, vol. 72, no. 2-6, pp. 195-200, 2006.

[13] H. Shokouhmand, F. Jam, and M. R. Salimpour, "Simulation of laminar flow and convective heat transfer in conduits filled with porous media using lattice Boltzmann Method," International Communications in Heat and Mass Transfer, vol. 36, no. 4, pp. 378-384, 2009.

[14] D. A. Nield, "Forced convection in a parallel plate channel with asymmetric heating," International Journal of Heat and Mass Transfer, vol. 47, no. 25, pp. 5609-5612, 2004.

[15] T. Seta, E. Takegoshi, and K. Okui, “Thermal lattice Boltzmann model for incompressible flows through porous media," Journal of Thermal Science and Technology, vol. 1, no. 2, pp. 90-100, 2006.

[16] S. Ergun, "Fluid flow through packed columns," Chemical Engineering Progress, vol. 48, no. 2, pp. 89-94, 1952.

[17] W. M. Kays and M. E. Crawford, Solutions Manual, Convective Heat and Mass Transfered, McGraw-Hill, New York, NY, USA, 3rd edition, 1993. 


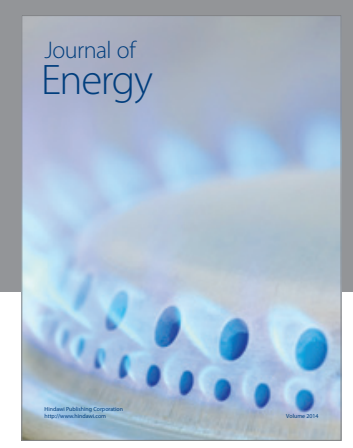

Journal of

Industrial Engineering
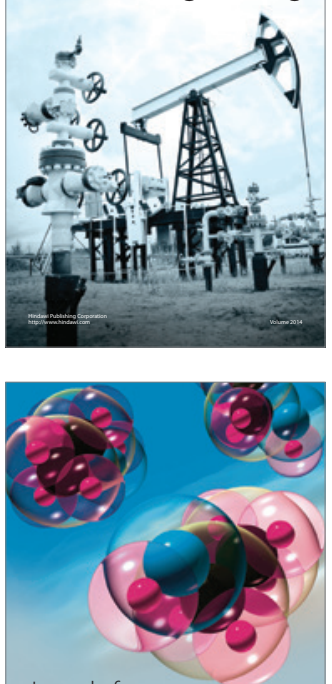

Fuels
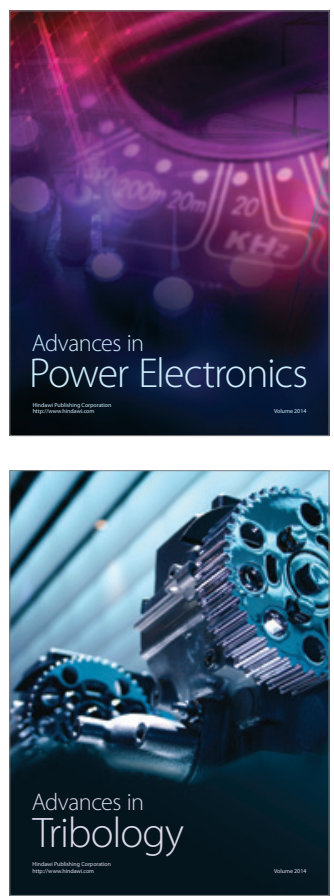

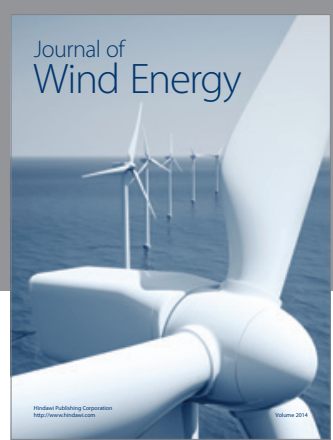

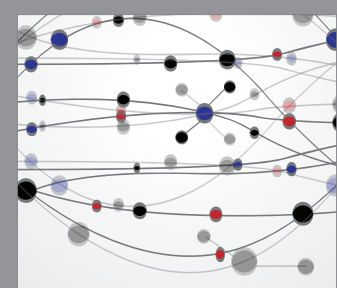

The Scientific World Journal

Submit your manuscripts at http://www.hindawi.com

Journal of

Structures
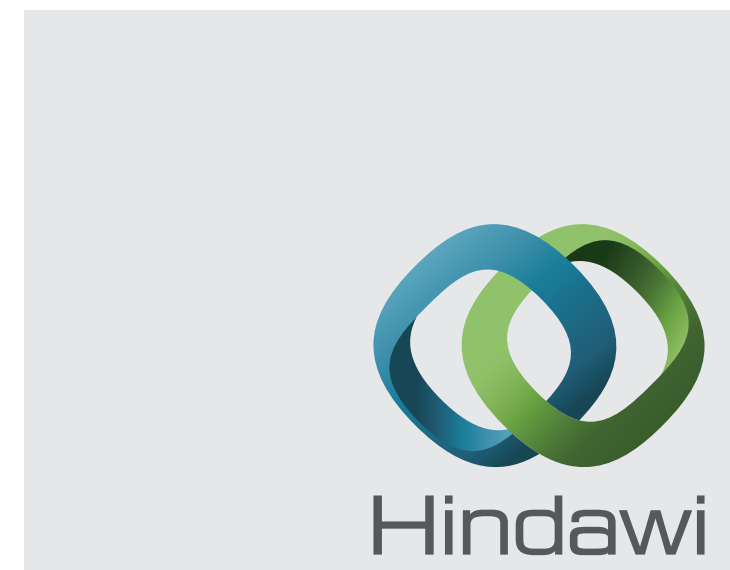

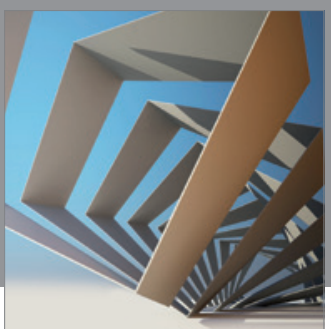

Rotating

Machinery
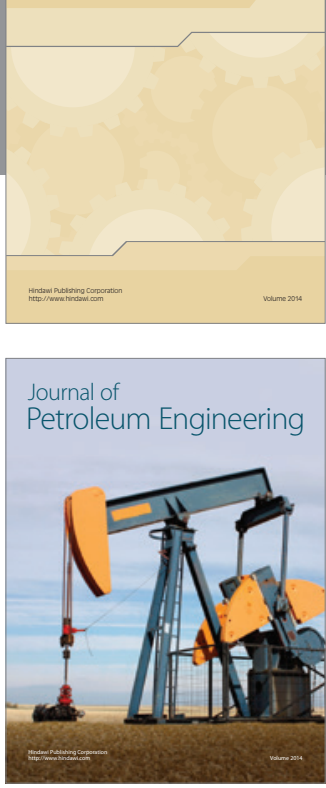

Journal of

Solar Energy
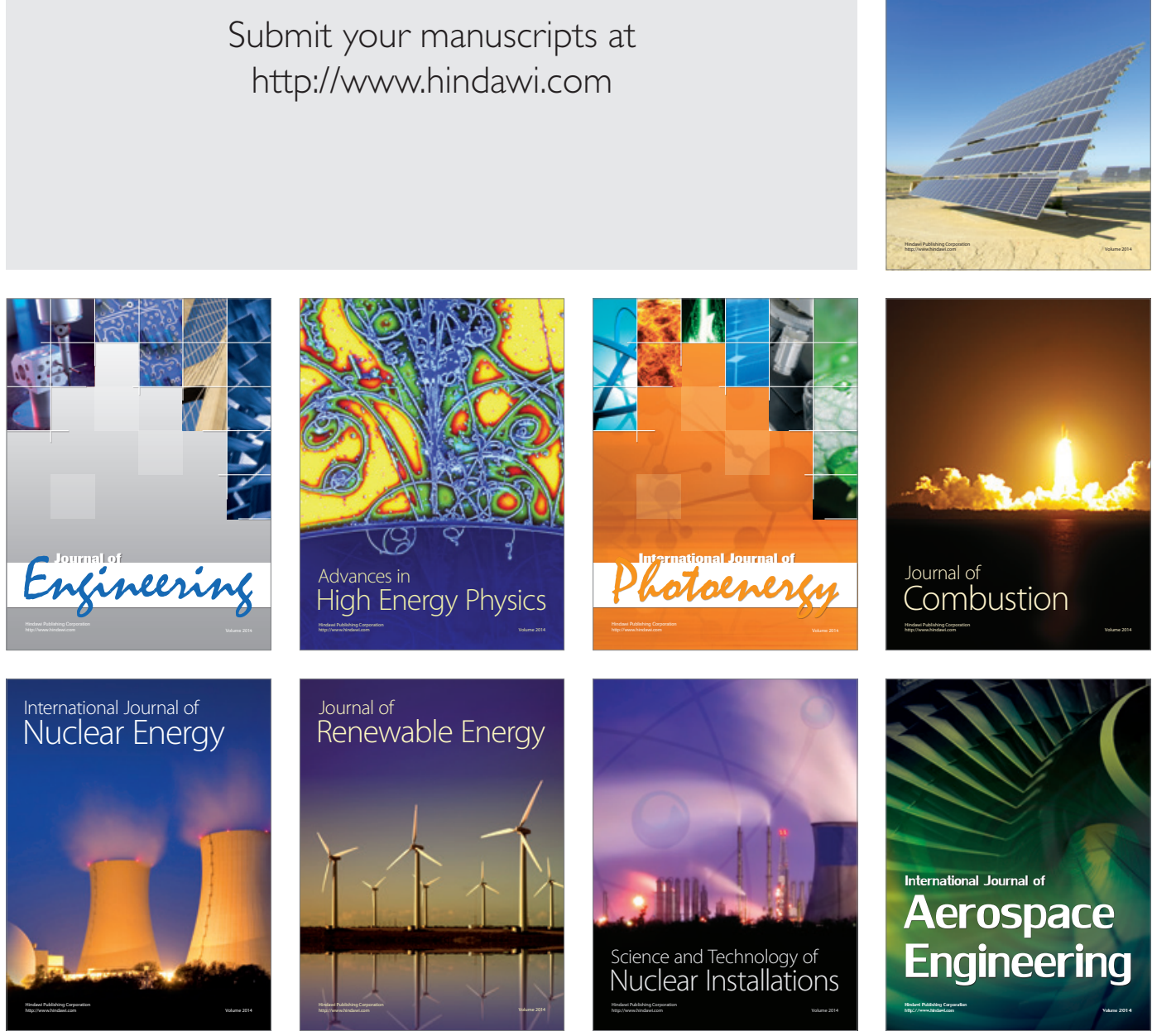